\title{
Overexpression of the small GTPase Arl4D suppresses adipogenesis
}

\author{
JIAHUA YU ${ }^{1,2}$, SUN-O KA ${ }^{1}$, KANG-BEOM KWON ${ }^{3}$, SANG-MYEONG LEE ${ }^{4}$, \\ JIN-WOO PARK ${ }^{1}$ and BYUNG-HYUN PARK ${ }^{1}$
}

\begin{abstract}
${ }^{1}$ Department of Biochemistry, Research Institute for Endocrine Sciences, and Diabetes Research Center, Chonbuk National University Medical School, Jeonju, Jeonbuk 561-756, Republic of Korea; ${ }^{2}$ Department of Radiobiology, School of Radiation Medicine and Public Health, Soochow University, Suzhou 215123, P.R. China; ${ }^{3}$ Department of Physiology, School of Oriental Medicine, Wonkwang University, Iksan, Jeonbuk 570-749, Republic of Korea; ${ }^{4}$ Division of Biotechnology, College of Environmental and Bioresource Sciences, Chonbuk National University, Iksan, Jeonbuk 570-752, Republic of Korea
\end{abstract}

Received May 27, 2011; Accepted June 29, 2011

DOI: 10.3892/ijmm.2011.751

\begin{abstract}
Arl4D is a developmentally-regulated member of the ADP-ribosylation factor/ARF-like protein (ARF/Arl) family of Ras-related GTPases. Although Arl4 protein is reported to be expressed in adipose tissue, the function of Arl4D is unknown. To investigate the potential role of Arl4D in adipogenesis, we examined Arl4D expression during adipocyte differentiation and the effects of Arl4D overexpression on adipogenesis. Arl4D protein increased early in adipogenesis, with the highest expression at $4 \mathrm{~h}$ after adipogenesis initiation, followed by a decrease thereafter. Overexpression of Arl4D in 3T3-L1 cells potently inhibited their ability to differentiate and accumulate lipid, and reduced the expression of adipogenic genes. Furthermore, treatment with valproic acid, an Arl4D inducer, suppressed adipogenesis. These results suggest that rapid reduction of Arl4D is required for adipogenesis to proceed.
\end{abstract}

\section{Introduction}

Obesity results from excess white adipose tissue, which can be caused by both adipocyte hyperplasia and hypertrophy (1). Hypertrophy is caused by excess triacylglyceride (TG) accumulation in adipocytes, while hyperplasia results from the differentiation of adipocytes from preadipocytes (2). The molecular mechanisms of adipocyte differentiation have been extensively studied (3). Adipogenesis is regulated by a complex network of transcription factors, with numerous activators,

Correspondence to: Dr Byung-Hyun Park, Department of Biochemistry, Chonbuk National University Medical School, San 2-20, Geumam-dong, Dukjin-gu, Jeonju, Jeonbuk 561-756, Republic of Korea

E-mail: bhpark@jbnu.ac.kr

Key words: Ar14D, adipogenesis, 3T3-L1, valproic acid co-activators, and repressors $(3,4)$. CCAAT/enhancer binding proteins (C/EBPs) and the peroxisome proliferator-activated receptor (PPAR) $\gamma$ are critical transcription factors in adipogenesis regulation. $\mathrm{C} / \mathrm{EBP} \beta$ and $\mathrm{C} / \mathrm{EBP} \delta$ are expressed in early adipogenesis, and they regulate the process by enhancing the subsequent expression of $\mathrm{C} / \mathrm{EBP} \alpha$ and PPAR $\gamma$ genes, which are involved in adipocyte maturation $(5,6)$.

ADP-ribosylation factors (ARFs) are a subfamily of the Ras-related GTPases. GTP binding translocates ARFs from the cytoplasm to the plasma membrane for interaction with a large number of effectors (7,8). ARF-like (Arl) proteins are evolutionarily conserved and share $40-60 \%$ amino acid identity with ARF proteins (9). Cellular processes regulated by Arl proteins include $\beta$-tubulin folding (10), vesicle transport (11), neurogenesis and somitogenesis during embryonic development, and the early stages of spermatogenesis in adults (12). To date, about 20 members of the Arl proteins have been identified in various species. Among the Arl proteins, Arl4 is particularly interesting because of its differential expression in 3T3-L1 cells: it is abundant in differentiated 3T3-L1 cells but undetectable in undifferentiated cells (13). Arl4A, Arl4C, and Arl4D are three closely related Arl4 proteins that appear to have recently diverged (14). To date, little is known about the function of Arl4D. It is localized in nuclei and nucleoli, and its expression is developmentally regulated during embryogenesis (12). Recent studies have shown that Arl4D, through binding to cytohesin-2, modulates actin remodeling and neurite outgrowth $(15,16)$. However, no studies have addressed its role in adipose tissue. Here we show that Arl4D expression is transiently increased in early adipogenesis, and that overexpression of Arl4D suppresses adipogenesis. This suggests that Arl4D functions as a gatekeeper of adipogenesis.

\section{Materials and methods}

Cell culture. 3T3-L1 cells were cultured in Dulbecco's modified Eagle's medium (DMEM) supplemented with $10 \%$ fetal bovine serum (FBS), $100 \mu \mathrm{g} / \mathrm{ml}$ streptomycin and $100 \mathrm{U} / \mathrm{ml}$ of penicillin in a humidified atmosphere of $5 \% \mathrm{CO}_{2} / 95 \%$ air at 
Table I. Sequences and accession numbers for primers used in real-time RT-PCR.

\begin{tabular}{llc}
\hline Gene & \multicolumn{1}{c}{ Sequences for primers } & Accession no. \\
\hline Arl4D & F: CAGAGGCCAAGAAGAGGAGA & NM025404 \\
& R: ACGAGTCGGTGTCTGGTTG & NM024406 \\
FP2 & R: TGGCTTTCTCACCTGGAAGA & NM007988 \\
FAS & F: TGATGTGGAACACAGCAAGG & NM008509 \\
LPL & R: GGCTGTGGTGACTCTTAGTGATAA & NM010719 \\
HSL & F: GGACGGTAACGGGAATGTATGA & R: TGACATTGGAGTCAGGTTCTCTCT \\
Pref-1 & F: GGAGCACTACAAACGCAACGA & N: TCGGCCACCGGTAAAGAG \\
& F: GCTGGGACGGGAAATTCTG & NM0052 \\
GAPDH & F: TCCAGGTCCACGCAAGTTC & \\
& R: TTGATGGCAACAATCTCCAC &
\end{tabular}

F, forward; R, reverse.

$37^{\circ} \mathrm{C}$. For 3T3-L1 differentiation, confluent cells were treated with differentiation medium [MDI, DMEM with $10 \% \mathrm{FBS}$, $1 \mu \mathrm{M}$ dexamethasone, $10 \mu \mathrm{g} / \mathrm{ml}$ insulin, and $0.5 \mathrm{mM}$ isobutylmethylxanthine (IBMX)] for 2 days. The medium was then replaced with standard medium containing $10 \mu \mathrm{g} / \mathrm{ml}$ insulin and changed every other day for 6 days (17).

Oil Red $O$ staining. Cells were washed twice with PBS and fixed with $10 \%$ formaldehyde for $2 \mathrm{~h}$ at room temperature. After washing once with $60 \%$ isopropanol, cells were stained for $1 \mathrm{~h}$ at room temperature with filtered Oil Red O/60\% isopropanol. Cells were washed once with $60 \%$ isopropanol, and once with distilled water. Stained adipocytes were observed by light microscopy. Cellular triglyceride (TG) content was assayed using a TG assay kit (Sigma, St. Louis, MO).

Isolation of the stromal vascular fraction. The stromal vascular fraction (SVF) of the adipose tissue was obtained as previously described (18). Briefly, epididymal adipose tissue collected from male C57BL/6 mice was cut into small pieces and suspended in Krebs-Ringer bicarbonate buffer (119 mM NaCl, $4.74 \mathrm{mM} \mathrm{KCl,} 1.19$ mM KH $\mathrm{PO}_{4}, 1.19 \mathrm{mM}$ $\mathrm{MgSO}_{4}, 20 \mathrm{mM} \mathrm{NaHCO}, 10 \mathrm{mM}$ HEPES, $2.54 \mathrm{mM} \mathrm{CaCl}$, $\mathrm{pH}$ 7.4) containing 3.5\% BSA, $6 \mathrm{mM}$ glucose, and $1 \mathrm{mg} / \mathrm{ml}$ type II collagenase (Sigma). The tissue was placed in a shaking water bath at $37^{\circ} \mathrm{C}$ with continuous agitation for $60 \mathrm{~min}$. Following digestion, material was filtered through a $200-\mu \mathrm{m}$ pore size nylon mesh (Spectrum Laboratories, Inc., Rancho Dominguez, CA) to remove the undigested tissue and the filtrate was centrifuged for $10 \mathrm{~min}$ at $500 \mathrm{x}$ g at room temperature. The supernatant containing mature adipocytes was collected as the adipocyte fraction and the pellet was collected as the SVF. All experimental protocols were approved by the Institutional Animal Care and Use Committee of Chonbuk National University.
Western blot analysis. Cells were washed twice with ice-cold PBS and lysed with the M-PER mammalian protein extraction reagent (Pierce Biotechnology, Rockford, IL) for $20 \mathrm{~min}$ on ice, and $10 \mu \mathrm{g}$ of homogenate protein were separated by SDS-PAGE and transferred to PVDF membrane. After blocking with 5\% BSA, blots were probed with primary antibodies against Arl4D, PPAR $\gamma, \beta$-actin (Santa Cruz Biotechnology, Santa Cruz, CA), $\mathrm{C} / \mathrm{EBP} \alpha, \mathrm{C} / \mathrm{EBP} \beta$ or $\mathrm{C} / \mathrm{EBP} \delta$ (Cell Signaling Technology, Danvers, MA). Horseradish peroxidase-conjugated IgG (Santa Cruz Biotechnology) was used as a secondary antibody. Protein levels were visualized using enhanced chemiluminescence by LAS-1000 (Fujifilm, Japan).

RNA isolation and real-time $R T-P C R$. Total-RNA was extracted from 3T3-L1 adipocytes using TRIzol reagent (Invitrogen, Carlsbad, CA). RNA was precipitated with isopropanol and dissolved in diethylpyrocarbonate-treated distilled water. Total-RNA $(2 \mu \mathrm{g})$ was treated with RNase-free DNase (Invitrogen), and first-strand cDNA was generated using oligo(dT)-adaptor primers and reverse transcriptase (Takara, Japan). Specific primers (Table I) were designed using Primer Express software (Applied Biosynthesis, Foster City, CA). GAPDH was used as the invariant control. Real-time RT-PCR mixtures consisted of $10 \mathrm{ng}$ reverse-transcribed total-RNA, $200 \mathrm{nM}$ forward and reverse primers, and 2X PCR master mixture in a final volume of $10 \mu \mathrm{l}$. Reactions were carried out in 384-well plates using the ABI PRISM 7900HT Sequence Detection System (Applied Biosystems). All reactions were conducted in triplicate.

Isolation of mouse Arl4D cDNA. Total-RNA was prepared from mouse liver tissue, reverse transcribed to cDNA and used to amplify Arl4D cDNA. Arl4D cDNA was cloned by Phusion high-fidelity DNA polymerase (New England Biolabs, Ipswich, MA) with forward primer, CACCATGGGGAACCACTTG, 
A

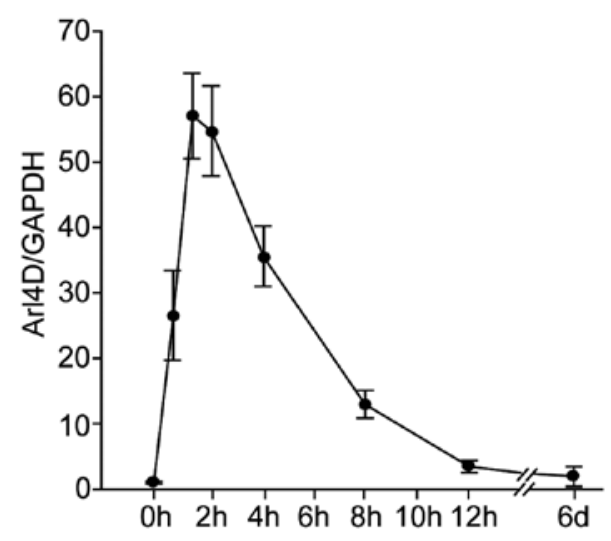

C

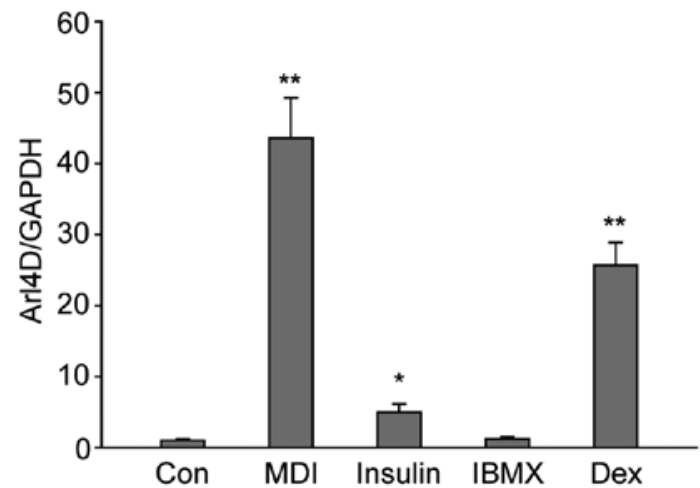

B
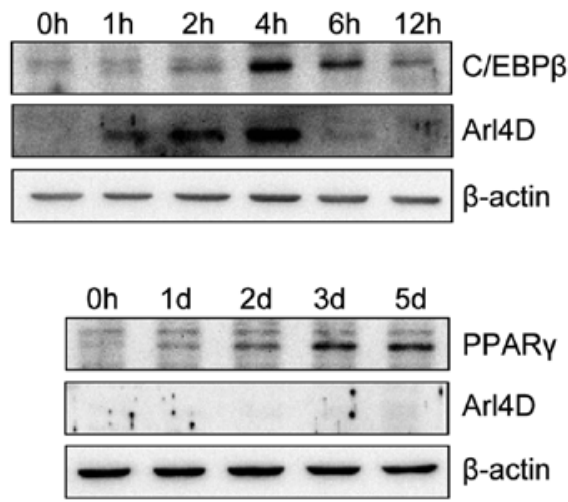

D

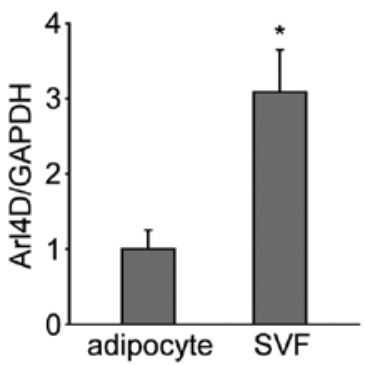

Figure 1. Arl4D expression during differentiation of 3T3-L1 cells. Two-day postconfluent 3T3-L1 preadipocytes were incubated with differentiation medium (MDI) and the total cellular RNA and protein samples were obtained at the indicated times. (A) Arl4D gene expression during adipogenesis. Arl4D mRNA levels were determined by real-time RT-PCR and normalized to GAPDH mRNA. Results from four independent experiments are presented as the mean \pm SEM. (B) Cellular Arl4D, C/EBP $\beta$, and PPAR $\gamma$ protein levels during adipogenesis. Total cell extracts were used for Western blot analyses. (C) 3T3-L1 cells were treated with MDI, insulin, isobutylmethylxanthine (IBMX) or desamethasone (Dex) for $2 \mathrm{~h}$ as indicated. Arl4D expression was quantified by real-time RT-PCR. ${ }^{*} \mathrm{P}<0.05 ;{ }^{* *} \mathrm{P}<0.01$ vs. control. (D) Arl4D expression in the adipocyte fraction and the stromal vascular fraction (SVF). Each value is the mean \pm SEM of five independent experiments. ${ }^{*}<0.05$ vs. adipocytes.

and reverse primer, CCGTCTCTTCTTGCTCGAC according the manufacturer's instructions. PCR reactions were: $98^{\circ} \mathrm{C}$ for $30 \mathrm{sec}, 32$ cycles at $98^{\circ} \mathrm{C}$ for $10 \mathrm{sec}, 60^{\circ} \mathrm{C}$ for $30 \mathrm{sec}$ and $72^{\circ} \mathrm{C}$ for $2 \mathrm{~min}$ and $72^{\circ} \mathrm{C}$ for $7 \mathrm{~min}$. PCR products were monitored by agarose gel electrophoresis. Positive clones were examined by sequencing.

Cell transduction with recombinant lentiviruses. Mouse Arl4D cDNA was cloned into the pENTR TOPO vector (Invitrogen). Site-specific recombination between entry vectors and the lentiviral destination vector (pLenti6.3/V5-DEST) used LR Clonase II (Invitrogen). An Arl4D lentivirus (Lenti-Arl4D) was generated in a 293FT cell line by ViraPower HiPerform Lentiviral Expression kit (Invitrogen). 3T3-L1 cells were transduced with lentivirus by $10 \mu \mathrm{g} / \mathrm{ml}$ polybrene and cells stably expressing Arl4D were selected using $10 \mu \mathrm{g} / \mathrm{ml}$ blasticidin for 2 weeks. Lenti-LacZ, which expresses $\beta$-galactosidase, was used as a control. Both Arl4D and $\beta$-galactosidase were expressed as C-terminal V5 fusion proteins.

Statistical analysis. All results are expressed as the mean \pm standard error of the mean (SEM), and the difference between groups was calculated using the Student's t-test. Differences with $\mathrm{P}<0.05$ were considered statistically significant.

\section{Results}

Expression of Arl4D during mouse 3T3-L1 differentiation. To analyze the expression pattern of Arl4D during adipocyte differentiation, RNA was isolated at different times during adipocyte differentiation, and gene expression measured by real-time RT-PCR. Arl4D mRNA was not detectable in either preadipocytes or mature adipocytes. However, a transient increase in Arl4D gene expression occurred early in adipogenesis (Fig. 1A). The Arl4D protein level peaked at $4 \mathrm{~h}$ after adipogenesis initiation, then quickly decreased to undetectable level at $12 \mathrm{~h}$. This indicates that Arl4D may be involved in early differentiation (Fig. 1B).

$\mathrm{C} / \mathrm{EBP} \beta$ and $\mathrm{C} / \mathrm{EBP} \delta$ are key transcription factors that act early in adipogenesis to activate other transcription factors $(5,6)$. C/EBP $\beta$ protein was expressed early in adipogenesis, with expression peaking at $4 \mathrm{~h}$ after adipogenesis initiation, then gradually decreasing (Fig. 1B). PPAR $\gamma$ protein, a key transcription factor in middle-late adipogenesis, was expressed at a low level early in adipogenesis, then increased 2 days after the initiation of adipocyte differentiation, and continued to increase thereafter (Fig. 1B).

Differentiation is triggered by a combination of insulin, dexamethasone, and IBMX, each of which activates complex 
A

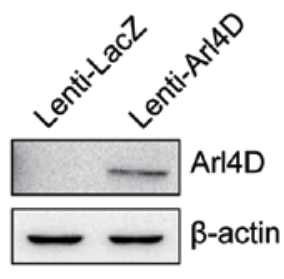

B

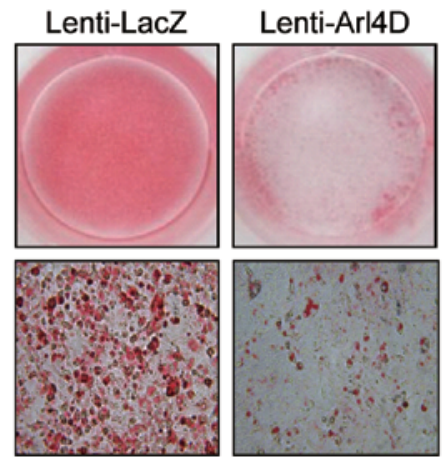

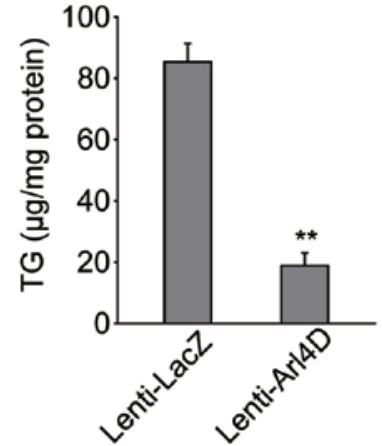

C

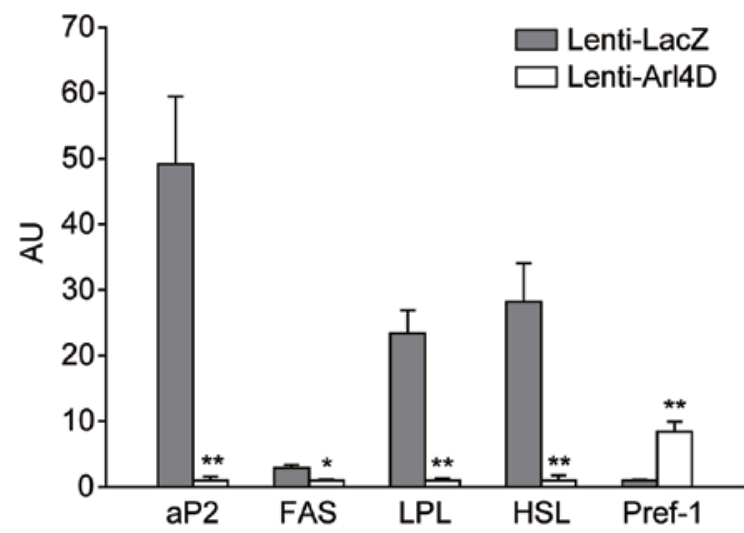

Figure 2. Overexpression of Arl4D in 3T3-L1 cells impairs adipogenesis. Arl4D was overexpressed in 3T3-L1 preadipocytes using a lentivirus. (A) Western blot analysis of Arl4D. (B) $\beta$-galactosidase or Arl4D overexpressing 3T3-L1 cells were grown in differentiation medium (MDI) for the first 2 days. Eight days after initiating differentiation, triglyceride content (TG) was visualized by Oil Red O staining and high magnification of cells. Cells were harvested and TG contents were measured. (C) mRNA levels of adipogenic marker genes aP2, fatty acid synthase (FAS), lipoprotein lipase (LPL) and hormone-sensitive lipase (HSL), and the preadipocyte marker gene Pref-1 were measured by real-time RT-PCR and normalized to GAPDH. Each value is the mean \pm SEM of four independent experiments. ${ }^{*} \mathrm{P}<0.05 ;{ }^{* *} \mathrm{P}<0.01$ vs. Lenti-LacZ.

downstream signaling. To identify the signaling pathways affecting Arl4D expression, we cultured post-confluent 3T3-L1 cells under different differentiation conditions. As shown in Fig. 1C, Arl4D expression was primarily induced by dexamethasone.

In white adipose tissue, Arl4D was more abundantly expressed in the SVF-containing preadipocytes than in the mature adipocyte fraction (Fig. 1D). This suggests that Arl4D may maintain adipose tissue mass in vivo.

Inhibition of adipogenesis through overexpression of Arl4D. To determine whether Arl4D regulates adipocyte differentiation, 3T3-L1 cells were infected with a Lenti-Arl4D to establish stable cells. Overexpression of Arl4D was demonstrated by Western blot analysis (Fig. 2A). Cell viability and mitotic clonal expansion were not altered by Arl4D overexpression (data not shown). Control virus-infected cells underwent normal MDI-induced adipogenesis and TG accumulation. In contrast, Arl4D overexpression led to marked inhibition of TG accumulation as shown by both Oil Red $\mathrm{O}$ staining and direct measurement of TG content (Fig. 2B). Total-RNA was extracted on the last day of differentiation (Day 8) and adipogenic gene expression profiles were generated by real-time RT-PCR (Fig. 2C). Consistent with the lipid accumulation data, mRNA levels of adipogenic marker genes such as adipocyte-specific lipid binding protein (aP2), fatty acid synthase (FAS), lipoprotein lipase (LPL) and hormonesensitive lipase (HSL) were repressed by Arl4D overexpression. Preadipocyte factor-1 (Pref-1), a preadipocyte marker that normally disappears during adipocyte maturation, was expressed at 8.4-fold over the control. Together, these data clearly indicate that continued Arl4D overexpression inhibited adipogenesis.

Regulation of adipogenic transcription factors expression by Arl $4 D$. Next, we determined the part of the transcriptional cascade that is affected by Arl4D during adipogenesis. Arl4D overexpression caused a marked decrease in $\mathrm{C} / \mathrm{EBP} \alpha$ and PPAR $\gamma$ expression, whereas $\mathrm{C} / \mathrm{EBP} \beta$ and $\mathrm{C} / \mathrm{EBP} \delta$ were unchanged during adipogenesis (Fig. 3). These results suggest that Arl4D does not affect early adipogenesis, but interferes with the induction of later transcription factors, leading to impaired adipogenesis.

Inhibition of adipogenesis by valproic acid. Because Arl4D overexpression reduced adipogenesis, we examined whether valproic acid, an Arl4D inducer, modulated adipogenesis. We first confirmed the induction of Arl4D in 3T3-L1 cells after treatment with valproic acid (Fig. 4A). Similar to the results obtained using Arl4D-overexpressing 3T3-L1 cells, treatment with valproic acid inhibited lipid accumulation in a concentration-dependent manner (Fig. 4B). 
Lenti-LacZ

Oh 2h 4h 8 h12h 1d 2d 3d 4d 5d

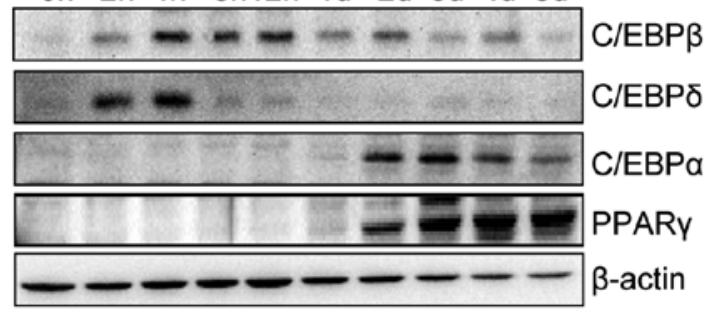

Lenti-Arl4D

Oh $2 \mathrm{~h} 4 \mathrm{~h} 8 \mathrm{~h} 12 \mathrm{~h} 1 \mathrm{~d} 2 \mathrm{~d} 3 \mathrm{~d} 4 \mathrm{~d} 5 \mathrm{~d}$

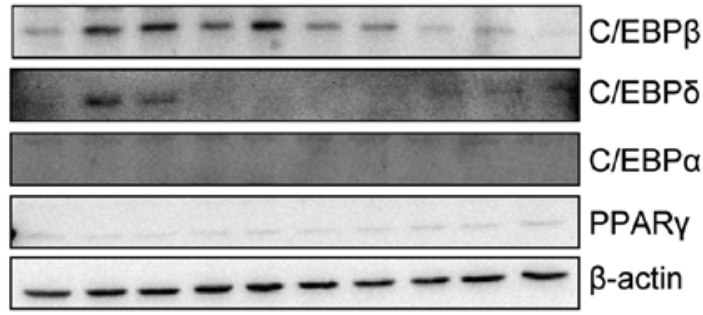

Figure 3. Overexpression of Arl4D in 3T3-L1 cells reduces expression of late stage transcription factors. $\beta$-galactosidase- or Arl4D-overexpressing 3T3-L1 cells underwent adipogenesis for the indicated times. Expression levels of C/EBP $\beta, C / E B P \delta, C / E B P \alpha$ and PPAR $\gamma$ were determined by Western blot analysis.

A

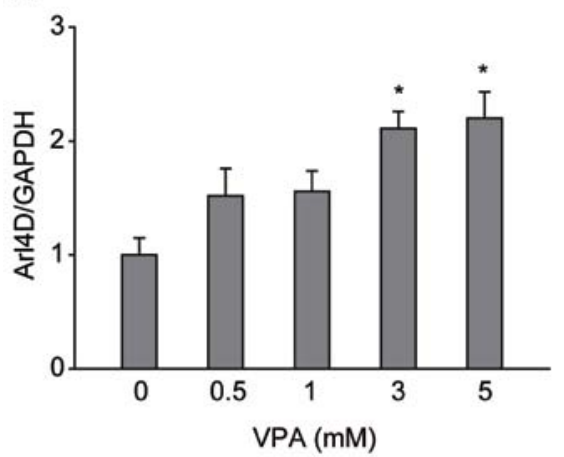

B

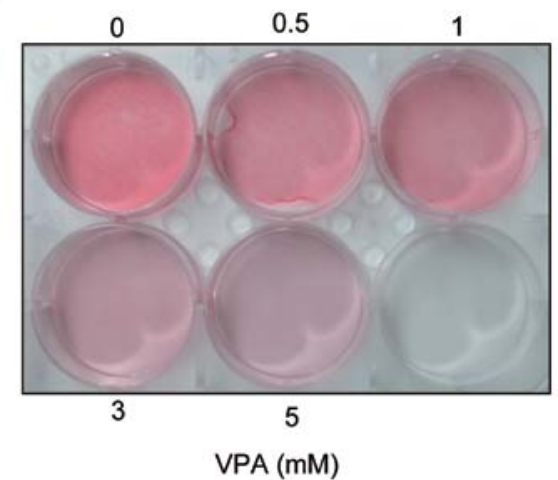

Figure 4. Valproic acid inhibits adipogenesis. (A) 3T3-L1 cells were treated with the indicated concentrations of valproic acid (VPA) for 2 days and Arl4D mRNA levels were determined by real-time RT-PCR. (B) Representative Oil Red O staining of 3T3-L1 cells treated with valproic acid. Values are the mean \pm SEM of four independent experiments. ${ }^{*} \mathrm{P}<0.05$ vs. control.

\section{Discussion}

This study investigated the expression pattern of Arl4D and conducted gain-of-function studies during adipogenesis. We show for the first time that Arl4D is a negative regulator of adipogenesis, based on the following findings: i) Arl4D expression endogenously increased in 3T3-L1 preadipocytes within a few hours of exposure to adipogenic stimuli; ii) overexpression of Arl4D delayed adipocyte differentiation, reduced intracellular TG accumulation and suppressed adipogeneic gene expression and iii) treatment of 3T3-L1 cells with valproic acid, an Arl4D inducer, delayed adipogenesis.

After peaking around $4 \mathrm{~h}$ after the beginning of differentiation, Arl4D protein level dramatically decreased to an undetectable level at $12 \mathrm{~h}$. This suggested that rapid reduction of Arl4D is required to relieve its inhibitory action on adipogenesis and allow adipogenesis to proceed. Mature adipocyte formation is hindered by Arl4D protein level remaining high by ectopic overexpression. Thus, Arl4D could be an important gatekeeper during adipocyte differentiation, thereby influencing lipid accumulation and adipocyte differentiation.

Sequential activation of transcription factors such as $\mathrm{C} /$ $\mathrm{EBP} \beta$ and $\mathrm{C} / \mathrm{EBP} \delta$ at an earlier stage, and $\mathrm{C} / \mathrm{EBP} \alpha$ and PPAR $\gamma$ at later stages, regulates the coordinated expression of several adipogenic factors $(5,6)$. In this study, we found that Arl4D overexpression suppressed expression of $\mathrm{C} / \mathrm{EBP} \alpha$ and $\operatorname{PPAR} \gamma$, but not $\mathrm{C} / \mathrm{EBP} \beta$ and $\mathrm{C} / \mathrm{EBP} \delta$. These findings suggested that Arl4D acts distally to $\mathrm{C} / \mathrm{EBP} \beta$ and $\mathrm{C} / \mathrm{EBP} \delta$ expression and proximally to $\mathrm{C} / \mathrm{EBP} \alpha$ and PPAR $\gamma$ expression in differentiation. Due to the fact that Arl4D is found in both the nucleus and the plasma membrane, the level at which Arl4D regulates the expression of $\mathrm{C} / \mathrm{EBP} \alpha$ and PPAR $\gamma$ remains to be discovered.

It is of interest to examine the mechanism by which Arl4D influences adipogenesis in future research. Reports characterizing Arl4D function provide some clues to its mechanism of action. Arl4D plays a role in activating cytohesin-2/ARNO and Arf6, thus acting as a novel regulator of actin reorganization $(15,19)$. Interestingly, cytohesin-2/ARNO and Arf6 control the migration of 3T3-L1 preadipocytes (20). During adipogenesis, adipocytes morphologically change from fibroblastic cells to round, lipid-laden cells. The actin cytoskeleton is known to regulate cell morphology (21). Adipocyte differentiation is reported to result in the conversion of filamentous actin (F-actin) from stress fibers to cortical actin structures (22). Indeed, several reports indicate a close relationship between cytoskeletal changes and adipocyte differentiation. For example, genetic and pharmacological inhibition of Rho-associated kinase, downstream of the small GTPase Rho, re-organizes the actin cytoskeleton and enhances adipogenesis through the up-regulation of adipogenic transcription factors in 3T3-L1 cells (23). In addition, the expression of actin is down-regulated during adipocyte differentiation (24). These reports suggest that Arl4D may be involved in the alteration of the actin cytoskeleton at early adipocyte differentiation. Future studies on the influence of Arl4D on the cytoskeleton during adipogenesis will be of interest.

Finally, we used valproic acid, which is currently in clinical use for people with epilepsy. A previous report showed that Arl4D is induced by valproic acid in neuroblastoma cells and it regulates neuronal differentiation through interaction with 
cytohesin-2 and Arf6 (16). In this study, we observed increased expression of Arl4D by valproic acid in 3T3-L1 cells. In addition, valproic acid effectively reduced adipogenesis and adipogenic gene induction, confirming the function of Arl4D in adipogenesis regulation. These results are in agreement with previous reports showing that valproic acid inhibits adipogenesis in 3T3-L1 cells and human preadipocytes $(25,26)$. In those studies, the effects of valproic acid were explained through suppression of histone deacetylase activity. Here, we propose that Arl4D induction may be another way for valproic acid to block adipogenesis.

In conclusion, our data indicate an important role for Arl4D in adipocyte differentiation. Arl4D was specific to the initial phase of adipogenesis. Overexpression of Arl4D suppressed $\mathrm{C} / \mathrm{EBP} \alpha$ and PPAR $\gamma$ expression, leading to delayed adipocyte differentiation. With the increasing prevalence of obesity worldwide, researchers are actively seeking new antiobesity targets. This study shows a novel function for Arl4D in adipose tissue and provides new insights into the regulation of adipocyte differentiation. The expression of Arl4D in adipocytes could be a potential target for the treatment of obesity and type II diabetes.

\section{Acknowledgements}

This study was supported by the National Research Foundation of Korea Grant funded by the Korean Government (no. 20100029463).

\section{References}

1. Unger RH: Minireview: weapons of lean body mass destruction: the role of ectopic lipids in the metabolic syndrome. Endocrinology 144: 5159-5165, 2003.

2. Spiegelman BM and Flier JS: Obesity and the regulation of energy balance. Cell 104: 531-543, 2001.

3. Farmer SR: Transcriptional control of adipocyte formation. Cell Metab 4: 263-273, 2006.

4. Lefterova MI and Lazar MA: New developments in adipogenesis. Trends Endocrinol Metab 20: 107-114, 2009.

5. Rosen ED, Walkey CJ, Puigserver P and Spiegelman BM: Transcriptional regulation of adipogenesis. Genes Dev 14: 1293-1307, 2000.

6. Tontonoz P, Hu E and Spiegelman BM: Stimulation of adipogenesis in fibroblasts by PPAR $\gamma 2$, a lipid-activated transcription factor. Cell 79: 1147-1156, 1994 .

7. Gillingham AK and Munro S: The small G proteins of the Arf family and their regulators. Annu Rev Cell Dev Biol 23: 579-611, 2007.

8. Spang A, Shiba Y and Randazzo PA: Arf GAPs: gatekeepers of vesicle generation. FEBS Lett 584: 2646-2651, 2010.
9. Burd CG, Strochlic TI and Gangi Setty SR: Arf-like GTPases: not so Arf-like after all. Trends Cell Biol 14: 687-694, 2004.

10. Bhamidipati A, Lewis SA and Cowan NJ: ADP ribosylation factor-like protein 2 (Arl2) regulates the interaction of tubulinfolding cofactor D with native tubulin. J Cell Biol 149: 1087-1096, 2000.

11. Lu L, Tai G and Hong W: Autoantigen Golgin-97, an effector of Arl1 GTPase, participates in traffic from the endosome to the trans-golgi network. Mol Biol Cell 15: 4426-4443, 2004.

12. Lin CY, Huang PH, Liao WL, et al: ARL4, an ARF-like protein that is developmentally regulated and localized to nuclei and nucleoli. J Biol Chem 275: 37815-37823, 2000.

13. Schurmann A, Breiner M, Becker W, et al: Cloning of two novel ADP-ribosylation factor-like proteins and characterization of their differential expression in 3T3-L1 cells. J Biol Chem 269: 15683-15688, 1994.

14. Li Y, Kelly WG, Logsdon JM Jr, et al: Functional genomic analysis of the ADP-ribosylation factor family of GTPases: phylogeny among diverse eukaryotes and function in C. elegans. FASEB J 18: 1834-1850, 2004.

15. Li CC, Chiang TC, Wu TS, Pacheco-Rodriguez G, Moss J and Lee FJ: ARL4D recruits cytohesin-2/ARNO to modulate actin remodeling. Mol Biol Cell 18: 4420-4437, 2007.

16. Yamauchi J, Miyamoto Y, Torii T, et al: Valproic acid-inducible Arl4D and cytohesin-2/ARNO, acting through the downstream Arf6, regulate neurite outgrowth in N1E-115 cells. Exp Cell Res 315: 2043-2052, 2009.

17. Yu J, Kwon KB, Park JW, Chae HJ and Park BH: Bax inhibitor-1 overexpression reduces the suppressive effect of calcium mobilizing agent on adipogenesis. Int J Biochem Cell Biol 41: 1739-1745, 2009.

18. Yu J, Yu HC, Kim KA, et al: Differences in the amount of lipolysis induced by atrial natriuretic peptide in small and large adipocytes. J Pept Sci 14: 972-977, 2008.

19. Hofmann I, Thompson A, Sanderson CM and Munro S: The Arl4 family of small $\mathrm{G}$ proteins can recruit the cytohesin Arf6 exchange factors to the plasma membrane. Curr Biol 17: 711-716, 2007.

20. Torii T, Miyamoto Y, Sanbe A, Nishimura K, Yamauchi J and Tanoue A: Cytohesin-2/ARNO, through its interaction with focal adhesion adaptor protein paxillin, regulates preadipocyte migration via the downstream activation of Arf6. J Biol Chem 285: 24270-24281, 2010

21. Jaffe AB and Hall A: Rho GTPases: biochemistry and biology. Annu Rev Cell Dev Biol 21: 247-269, 2005.

22. Kanzaki M and Pessin JE: Insulin-stimulated GLUT4 translocation in adipocytes is dependent upon cortical actin remodeling. $\mathrm{J}$ Biol Chem 276: 42436-42444, 2001.

23. Noguchi M, Hosoda K, Fujikura J, et al: Genetic and pharmacological inhibition of Rho-associated kinase II enhances adipogenesis. J Biol Chem 282: 29574-29583, 2007.

24. Spiegelman BM and Farmer SR: Decreases in tubulin and actin gene expression prior to morphological differentiation of 3T3 adipocytes. Cell 29: 53-60, 1982.

25. Catalioto RM, Maggi CA and Giuliani S: Chemically distinct HDAC inhibitors prevent adipose conversion of subcutaneous human white preadipocytes at an early stage of the differentiation program. Exp Cell Res 315: 3267-3280, 2009.

26. Lagace DC and Nachtigal MW: Inhibition of histone deacetylase activity by valproic acid blocks adipogenesis. J Biol Chem 279: 18851-18860, 2004. 